\title{
An $N=8$ Superaffine Malcev Algebra and Its $N=8$ Sugawara
}

\author{
H.L. Carrion, M. Rojas and F. Toppan \\ $C B P F-C C P$ \\ Rua Dr. Xavier Sigaud 150, cep 22290-180 Rio de Janeiro (RJ) \\ Brazil
}

\begin{abstract}
A supersymmetric affinization of the algebra of octonions is introduced. It satisfies a superMalcev property and is $N=8$ supersymmetric. Its Sugawara construction recovers, in a special limit, the non-associative $N=8$ superalgebra of Englert et al.

This paper extends to supersymmetry the results obtained by Osipov in the bosonic case.
\end{abstract}

E-mails: lenyj@cbpf.br, mrojas@cbpf.br, toppan@cbpf.br

CBPF-NF-011/01 


\section{Introduction.}

It is well-known that a connection exists (see e.g. [1]), between the division algebras of complex numbers, quaternions, octonions and the $N$-extended supersymmetries, for values $N=2,4,8$ respectively. However, the characterization of $N$-extended supersymmetries in terms of division algebras is not always worked out explicitly. This is especially true in the case of the octonions since, being both non-commutative and non-associative, they present peculiar difficulties.

In the present work we extend to the supersymmetric case the approach and results obtained by Osipov some years ago in a series of three papers concerning the affinization [2, 3] of the octonionic algebra and its Sugawara [2] construction.

We postpone to the Conclusions (where a list of possible applications of the results here obtained is furnished) the discussion about the motivations of our paper. In this Introduction we limit ourselves to summarize the main results of the present work.

The algebra of octonions is supersymmetrically affinized following a proposal made in [2, 3]. While in [2] the bosonic case only was considered, in [3] a superaffinization was proposed. The superaffinization introduced here however, formulas (111) and (14), differs from the one in [3]. Our formulation is manifestly supersymmetric, while the one of [3] is not.

Later we explicitly prove that the superaffine octonionic algebra (11) is superMalcev, i.e. it satisfies a graded version of the Malcev identity.

Despite being expressed in terms of manifestly $N=1$ superfields, the (11) superaffine algebra is $N=8$ supersymmetric. One way of seeing this is in consequence of the existence of a supersymmetric Sugawara construction given in formulas (16). This Sugawara realization is a generalization of both the Osipov's construction [2] in the purely bosonic case (all fermionic fields set equal to zero), as well as the $N=4$ S.C.A. Sugawara of reference [䧃, recovered when just the (superaffinized version of the) quaternionic subalgebra of the octonions is taken into account.

Unlike the purely bosonic [2] and the $N=4$ S.C.A. [4] Sugawaras, the Sugawara-induced fields (16) do not close a superalgebra due to the presence of extra-terms (dependent on fermionic fields associated to octonionic structure-constants not belonging to a quaternionic subalgebra). However, after a suitable limiting procedure is taken into account, a closed $N=8$ generalization of the Virasoro algebra (with one bosonic spin-2 field, 8 fermionic spin- $\frac{3}{2}$ and 7 bosonic spin-1 fields) is recovered. It corresponds to the so-called "Non-associative $N=8$ S.C.A." introduced for the first time by Englert et al. in reference [5]. Therefore, as a byproduct of our investigation concerning the superaffinization of the algebra of the octonions and its Sugawara realization, we found as a bonus its connection with a very remarkable and quite "mysterious" superconformal algebra appearing in the literature. The latter algebra is not of (super)Malcev type. This point will be commented in the text.

In order to obtain our results we made extensive use of the Thielemans' package for classical OPE computations with Mathematica. We developed our own special package to deal with octonions.

\section{Notations and preliminary results}

In this section the basic properties of the division algebra of the octonions, following [6], 
are reviewed. They are later used for our construction.

A generic octonion $x$ is expressed as $x=x_{a} \tau_{a}$ (throughout the text the convention over repeated indices, unless explicitly mentioned, is understood), where $x_{a}$ are real numbers while $\tau_{a}$ denote the basic octonions, with $a=0,1,2, \ldots, 7$.

$\tau_{0} \equiv \mathbf{1}$ is the identity, while $\tau_{\alpha}$, for $\alpha=1,2, \ldots, 7$, denote the imaginary octonions. In the following a Greek index is employed for imaginary octonions, a Latin index for the whole set of octonions (identity included).

The octonionic multiplication can be introduced through

$$
\tau_{\alpha} \cdot \tau_{\beta}=-\delta_{\alpha \beta} \tau_{0}+C_{\alpha \beta \gamma} \tau_{\gamma}
$$

with $C_{\alpha \beta \gamma}$ a set of totally antisymmetric structure constants which, without loss of generality, can be taken to be

$$
C_{123}=C_{147}=C_{165}=C_{246}=C_{257}=C_{354}=C_{367}=1 .
$$

and vanishing otherwise.

It is also convenient to introduce, in the seven-dimensional imaginary octonions space, a 4-indices totally antisymmetric tensor $C_{\alpha \beta \gamma \delta}$, dual to $C_{\alpha \beta \gamma}$, through

$$
C_{\alpha \beta \gamma \delta}=\frac{1}{6} \varepsilon_{\alpha \beta \gamma \delta \epsilon \zeta \eta} C_{\epsilon \zeta \eta}
$$

(the totally antisymmetric tensor $\varepsilon_{\alpha \beta \gamma \delta \epsilon \zeta \eta}$ is normalized so that $\varepsilon_{1234567}=+1$ ).

The octonionic multiplication is not associative since for generic $a, b, c$ we $\operatorname{get}\left(\tau_{a} \cdot \tau_{b}\right) \cdot \tau_{c} \neq$ $\tau_{a} \cdot\left(\tau_{b} \cdot \tau_{c}\right)$. However the weaker condition of alternativity is satisfied. This means that, for $a=b$, the associator

$$
\left[\tau_{a}, \tau_{b}, \tau_{c}\right] \equiv\left(\tau_{a} \cdot \tau_{b}\right) \cdot \tau_{c}-\tau_{a} \cdot\left(\tau_{b} \cdot \tau_{c}\right)
$$

is vanishing.

We further introduce the commutator algebra of octonions through

$$
\left[\tau_{a}, \tau_{b}\right]={ }_{\text {def }} \tau_{a} \cdot \tau_{b}-\tau_{b} \cdot \tau_{a}=f_{a b c} \tau_{c}
$$

$f_{a b c}$ are the structure constants and can be read from (1); we have $f_{a b c}=2 C_{a b c}$ (where $C_{a b c}$ coincides with $C_{\alpha \beta \gamma}$ for $a, b, c=1,2, \ldots, 7$ and is vanishing otherwise).

The above-defined commutator brackets [.,.] satisfy the two properties below, which make the commutator algebra a Malcev-type algebra

$$
\begin{aligned}
{[\mathbf{x}, \mathbf{x}] } & =0 \\
J(\mathbf{x}, \mathbf{y},[\mathbf{x}, \mathbf{z}]) & =[J(\mathbf{x}, \mathbf{y}, \mathbf{z}), \mathbf{x}]
\end{aligned}
$$

for any given triple $\mathbf{x}, \mathbf{y}, \mathbf{z}$ of octonions.

$J(\mathbf{x}, \mathbf{y}, \mathbf{z})$ is the Jacobian

$$
J(\mathbf{x}, \mathbf{y}, \mathbf{z})=[[\mathbf{x}, \mathbf{y}], \mathbf{z}]]+[[\mathbf{y}, \mathbf{z}], \mathbf{x}]+[[\mathbf{z}, \mathbf{x}], \mathbf{y}] .
$$

The second relation in (6) is in consequence of the alternativity property. 
Malcev algebras are a special generalization of the Lie algebras, obtained by relaxing the condition of the vanishing of the Jacobian.

\section{The supersymmetric affinization of the octonionic al- gebra.}

In this section we introduce the superaffinization of the commutator algebra of the octonions (5) or, in short, the superaffine octonionic algebra, which for later convenience will be denoted as $\widehat{\mathcal{O}}$-algebra. Since the commutator algebra of octonions (5) is not a Lie algebra, its superaffinization implies generalizing the concept of superaffinization of a given Lie algebra $\mathcal{G}$. However, the standard notion of superaffinization should be recovered when formulas are specialized to the subalgebra of quaternions, which is equivalent to the Lie algebra $\operatorname{sl}(2) \oplus o(2)$.

Given a Lie algebra $\mathcal{G}$ with generators $g_{i}$ and structure constants $f_{i j k}$, the superaffine Lie algebra $\widehat{\mathcal{G}}$ is introduced by associating a bosonic spin-1 field $j_{i}(x)$ and a spin- $\frac{1}{2}$ fermionic field $\psi_{i}(x)$ to each Lie algebra generator $g_{i}$. These fields can be thought as components of a single fermionic $N=1$ superfield $\Psi_{i}(X)=\psi_{i}(x)+\theta j_{i}(x)$, where $X \equiv(x, \theta)$ denotes a superspace coordinate and $\theta$ is a Grassmann variable satisfying $\theta^{2}=0$. The superaffine $\widehat{\mathcal{G}}$ algebra is introduced through the brackets

$$
\left\{\Psi_{i}(X), \Psi_{j}(Y)\right\}=f_{i j k} \Psi_{k}(Y) \delta(X, Y)+k \cdot \operatorname{tr}\left(g_{i} g_{j}\right) D_{Y} \delta(X, Y)
$$

In the formula above $X \equiv x, \theta$ and $Y \equiv y, \eta$ are superspace coordinates. $\delta(X, Y)$ is the supersymmetric delta-function

$$
\delta(X, Y)=\delta(x-y)(\theta-\eta)
$$

and $D_{Y}$ the supersymmetric derivative

$$
D_{Y}=\frac{\partial}{\partial \eta}+\eta \partial_{y}
$$

The last term in the r.h.s. of (8) corresponds to the central extension (in its absence we obtain the superloop algebra). The trace is taken in a given representation of $\mathcal{G}$ (let's say the adjoint). The formula (8) is manifestly supersymmetric since it is constructed with $N=1$ superfields and covariant quantities.

In the absence of the central extension, the construction of the superloop extension of the (5) octonionic algebra is straightforward. The construction of the central extension is however delicate. In this case it is no longer possible to introduce it by making reference to a matrix representation since no such representation exists for the non-associative algebra of the octonions. Our proposal consists in introducing the superaffine algebra $\widehat{\mathcal{O}}$, based on the fermionic superfields $\Psi_{a}(X), a=0,1,2, \ldots, 7$, through the position

$$
\left\{\Psi_{a}(X), \Psi_{b}(Y)\right\}=f_{a b c} \Psi_{c}(Y) \delta(X, Y)+k \cdot \Pi\left(\tau_{a} \cdot \tau_{b}\right) D_{Y} \delta(X, Y)
$$

where $\Pi\left(\tau_{a} \cdot \tau_{b}\right)$ denotes the projection over the identity $\mathbf{1}$ in the composition law.

Some comments are in order. With the above definition the correct (anti-)symmetry properties of the brackets are satisfied. The algebra is manifestly $N=1$ supersymmetric and, when 
the formulas are specialized to the quaternionic subalgebra, we recover the standard superaffinization commented above. Moreover the Osipov's bosonic construction is recovered when setting the fermionic fields equal to zero.

We therefore regard (11) as the correct superaffinization of the commutator algebra of the octonions. It should be noticed that a superaffinization has been proposed in [3] in terms of component fields. However the algebra introduced in [3] presents a central extension which fails to be manifestly supersymmetric, in contrast with the (11) algebra.

The superaffine $\widehat{\mathcal{O}}$ algebra is superMalcev, as a simple inspection can prove, i.e. it satisfies a $\mathbf{Z}_{2}$-graded extension of the Malcev properties, where the brackets are now $\mathbf{Z}_{2}$-graded and the Jacobian is replaced by a superJacobian. The $\epsilon_{\mathbf{x}}$ grading of the $\mathbf{x}$ field is either 0 or 1 according to the bosonic (respectively fermionic) character of $\mathbf{x}$. The graded brackets are defined as

$$
[\mathbf{x}, \mathbf{y}]=(-1)^{\epsilon_{\mathbf{x}} \epsilon_{\mathbf{y}}+1}[\mathbf{y}, \mathbf{x}]
$$

$(\mathbf{x}, \mathbf{y}$ denote graded-algebra elements like fields or superfields; in the following the gradedbrackets are denoted either as "[.,.]" or as "\{.,.\}" according to the convenience).

The superJacobian $J(\mathbf{x}, \mathbf{y}, \mathbf{z})$ is

$$
J(\mathbf{x}, \mathbf{y}, \mathbf{z})=(-1)^{\epsilon_{\mathbf{x}} \epsilon_{\mathbf{z}}}[\mathbf{x},[\mathbf{y}, \mathbf{z}]]+(-1)^{\epsilon_{\mathbf{y}} \epsilon_{\mathbf{x}}}[\mathbf{y},[\mathbf{z}, \mathbf{x}]]+(-1)^{\epsilon_{\mathbf{z}} \epsilon_{\mathbf{y}}}[\mathbf{z},[\mathbf{x}, \mathbf{y}]] .
$$

With this in mind, formulas (6) can be reinterpreted as conditions for the superMalcev property.

In component level, the superaffine $\widehat{\mathcal{O}}$ algebra reads as follows

$$
\begin{aligned}
\left\{\psi_{a}(x), \psi_{b}(y)\right\} & =k \delta_{a b} \delta(x-y), \\
\left\{\psi_{a}(x), j_{b}(y)\right\} & =f_{a b c} \psi_{c}(y) \delta(x-y), \\
\left\{j_{a}(x), j_{b}(y)\right\} & =k \delta_{a b} \partial_{y} \delta(x-y)+f_{a b c} j_{c}(y) \delta(x-y) .
\end{aligned}
$$

Here $j_{a}(x)$ ad $\psi_{a}(x)$ are real fields. It should be noticed that, for later convenience, in the above formulas the $\Psi_{0}(X)$ superfield has been associated with the $i \tau_{0}$ octonion. The real-valued $k$ is the central charge of the superaffine $\widehat{\mathcal{O}}$ algebra.

The proof that (14) satisfies the superMalcev conditions (6) consists in a straightforward check.

\section{The Sugawara construction and the $N=8$ Non - as- sociative S.C.A.}

In this Section we investigate the Sugawara construction performed with the superaffine fields entering the $\widehat{\mathcal{O}}$ algebra (14). We already know that the purely bosonic subalgebra admits a Sugawara described in [2], while it is possible to prove that the Sugawara associated to the quaternionic subalgebra (obtained when the octonionic coefficients $a, b$ are restricted to the values $0,1,2,3$ only) corresponds to the Sugawara of [4] which realizes the $N=4$ "minimal" Superconformal Algebra. Both such cases are recovered as a special limit of the Sugawara construction here described.

To be precise we are investigating here the possibility of a closed algebraic structure in the Enveloping Algebra of $\widehat{\mathcal{O}}$, thought as an algebra of real fields endowed with a non-Lie superPoisson bracket structure given by equation (14), assumed to satisfy a graded version of the 
Leibniz property, i.e.

$$
[\mathbf{x y}, \mathbf{z}]=\mathbf{x}[\mathbf{y}, \mathbf{z}]+(-1)^{\epsilon_{\mathbf{x}} \epsilon_{\mathbf{y}}} \mathbf{y}[\mathbf{x}, \mathbf{z}] \text {. }
$$

Specifically, we are looking for an $N=8$ extension of the Virasoro algebra, covariantly constructed with the imaginary octonions structure constants (labeled by the greek indices). This requires a spin-2 Virasoro-type field $T$ (scalar w.r.t imaginary octonions), a fermionic spin- $\frac{3}{2}$ field $Q$ (also scalar w.r.t imaginary octonions) and the extra seven spin- $\frac{3}{2}$ fields $Q_{\alpha}$, as well as the seven spin 1 bosonic currents $J_{\alpha}$.

This problem is well-defined and admits a complete solution which can be obtained via computer algebra. We indeed solved it by first rephrasing it in the language of classical OPE's, which allowed us to perform computations using Mathematica.

The final answer is the following. Unlike both the purely bosonic case and the $N=4 \mathrm{SCA}$ quaternionic subalgebra case, no closed algebraic structure can be found for a given finite value of $k$, the affine central charge entering (14). This is due to the presence of the extra-terms $(X \star)_{\ldots}(18)$, depending on the 4-indices structure constant $C_{\alpha \beta \gamma \delta}$ and the fermionic fields $\psi_{\alpha}$. All such terms are automatically vanishing for the two above-mentioned subalgebra cases.

However, a closed algebraic structure can be found after taking a well-definite limit for $k \rightarrow \infty$.

Indeed if we set

$$
\begin{aligned}
T & =\frac{1}{k^{2}}\left(j_{a} j_{a}+\psi_{a}^{\prime} \psi_{a}\right)+\frac{1}{k} j_{0}^{\prime}-\frac{2}{3 k^{3}} C_{\alpha \beta \gamma} \psi_{\alpha} \psi_{\beta} j_{\gamma}-\frac{1}{k^{4}} C_{\alpha \beta \gamma \delta} \psi_{\alpha} \psi_{\beta} \psi_{\gamma} \psi_{\delta}, \\
Q & =\frac{1}{k^{2}} \psi_{a} j_{a}+\frac{1}{k} \psi_{0}^{\prime}-\frac{2}{k^{3}} C_{\alpha \beta \gamma} \psi_{\alpha} \psi_{\beta} \psi_{\gamma}, \\
Q_{\alpha} & =\frac{1}{k^{2}}\left(\psi_{0} j_{\alpha}-\psi_{\alpha} j_{0}-C_{\alpha \beta \gamma} \psi_{\beta} j_{\gamma}\right)-\frac{1}{k} \psi_{\alpha}^{\prime}-\frac{2}{k^{3}} C_{\alpha \beta \gamma \delta} \psi_{\beta} \psi_{\gamma} \psi_{\delta}, \\
J_{\alpha} & =\frac{1}{k^{2}} \psi_{0} \psi_{\alpha}+\frac{1}{k} j_{\alpha}-\frac{1}{2 k} C_{\alpha \beta \gamma} \psi_{\beta} \psi_{\gamma}
\end{aligned}
$$

and simultaneously renormalize the Poisson brackets in (14) through

$$
\{., .\} \mapsto\{., .\}_{R}=\frac{k}{2}\{., .\}
$$

we obtain a closed algebraic structure, since all the extra-terms $(X \star)$.. which appear below are vanishing in this limit.

The result is in fact

$$
\begin{aligned}
\{T(x), T(y)\}_{R} & =-\frac{1}{2} \partial_{y}{ }^{3} \delta(x-y)+2 T(y) \partial_{y} \delta(x-y)+T^{\prime}(y) \delta(x-y), \\
\{T(x), Q(y)\}_{R} & =\frac{3}{2} Q(y) \partial_{y} \delta(x-y)+Q^{\prime}(y) \delta(x-y)+(X 1), \\
\left\{T(x), Q_{\alpha}(y)\right\}_{R} & =\frac{3}{2} Q_{\alpha}(y) \partial_{y} \delta(x-y)+Q_{\alpha}{ }^{\prime}(y) \delta(x-y)+(X 2)_{\alpha}, \\
\left\{T(x), J_{\alpha}(y)\right\}_{R} & =J_{\alpha}(y) \partial_{y} \delta(x-y)+J_{\alpha}{ }^{\prime}(y) \delta(x-y)+(X 3)_{\alpha}, \\
\{Q(x), Q(y)\}_{R} & =-\frac{1}{2} \partial_{y}{ }^{2} \delta(x-y)++\frac{1}{2} T(y) \delta(x-y)+(X 4), \\
\left\{Q(x), Q_{\alpha}(y)\right\}_{R} & =-J_{\alpha}(y) \partial_{y} \delta(x-y)-\frac{1}{2} J_{\alpha}{ }^{\prime}(y) \delta(x-y)+(X 5)_{\alpha},
\end{aligned}
$$




$$
\begin{aligned}
\left\{Q(x), J_{\alpha}(y)\right\}_{R}= & -\frac{1}{2} Q_{\alpha}(y) \delta(x-y)+(X 6)_{\alpha}, \\
\left\{Q_{\alpha}(x), Q_{\beta}(y)\right\}_{R}= & -\frac{1}{2} \delta_{\alpha \beta} \partial_{y}{ }^{2} \delta(x-y)+C_{\alpha \beta \gamma} J_{\gamma}(y) \partial_{y} \delta(x-y)+ \\
& +\frac{1}{2}\left(\delta_{\alpha \beta} T(y)+C_{\alpha \beta \gamma} J_{\gamma}{ }^{\prime}(y)\right) \delta(x-y)+(X 7)_{\alpha \beta}, \\
\left\{Q_{\alpha}(x), J_{\beta}(y)\right\}_{R}= & \frac{1}{2}\left(\delta_{\alpha \beta} Q(y)-C_{\alpha \beta \gamma} Q_{\gamma}(y)\right) \delta(x-y)+(X 8)_{\alpha \beta}, \\
\left\{J_{\alpha}(x), J_{\beta}(y)\right\}_{R}= & \frac{1}{2} \delta_{\alpha \beta} \partial_{y} \delta(x-y)-C_{\alpha \beta \gamma} J_{\gamma}(y) \delta(x-y)+(X 9)_{\alpha \beta},
\end{aligned}
$$

where the extra-terms $(X \star)_{\ldots}$, vanishing in the $k \rightarrow \infty$ limit, are explicitly given by $(X \star)_{\ldots} \equiv$ $(\widetilde{X \star}) \ldots(y) \delta(x-y)$, with

$$
\begin{aligned}
(\widetilde{X 1})= & -\frac{3}{2 k^{4}} C_{\alpha \beta \gamma \delta} \psi_{\alpha} \psi_{\beta} \psi_{\gamma} j_{\delta}, \\
(\widetilde{X 2})_{\alpha}= & \frac{12}{k^{5}} C_{\beta \gamma \delta} \psi_{0} \psi_{\alpha} \psi_{\beta} \psi_{\gamma} \psi_{\delta}-\frac{2}{k^{4}} C_{\alpha \beta \gamma \delta} \psi_{0} \psi_{\beta} \psi_{\gamma} j_{\delta}+\frac{12}{k^{5}} C_{\beta \gamma \delta \epsilon} \psi_{\alpha} \psi_{\beta} \psi_{\gamma} \psi_{\delta} \psi_{\epsilon}+ \\
& +\frac{4}{k^{4}} C_{\beta \gamma \delta} \psi_{\alpha} \psi_{\beta} \psi_{\gamma} j_{\delta}-\frac{6}{k^{4}} \delta_{\alpha \beta} j_{\beta} C_{\gamma \delta \epsilon} \psi_{\gamma} \psi_{\delta} \psi_{\epsilon}-\frac{6}{k^{4}} C_{\alpha \beta \gamma} \psi_{\beta} \psi_{\gamma} j_{\delta} \psi_{\delta}, \\
(\widetilde{X 3})_{\alpha}= & \frac{12}{k^{4}} C_{\beta \gamma \delta} \psi_{\alpha} \psi_{\beta} \psi_{\gamma} \psi_{\delta}-\frac{2}{k^{3}} C_{\alpha \beta \gamma \delta} \psi_{\beta} \psi_{\gamma} j_{\delta}, \\
(\widetilde{X 4})= & \frac{5}{2 k^{4}} C_{\alpha \beta \gamma \delta} \psi_{\alpha} \psi_{\beta} \psi_{\gamma} \psi_{\delta}, \\
(\widetilde{X 5})_{\alpha}= & \frac{2}{k^{4}} C_{\alpha \beta \gamma \delta} \psi_{0} \psi_{\beta} \psi_{\gamma} \psi_{\delta}-\frac{6}{k^{4}} C_{\beta \gamma \delta} \psi_{\alpha} \psi_{\beta} \psi_{\gamma} \psi_{\delta}-\frac{1}{3 k^{3}} C_{\alpha \beta \gamma \delta} \psi_{\beta} \psi_{\gamma} j_{\delta}, \\
(\widetilde{X 6})_{\alpha}= & -\frac{7}{3 k^{3}} C_{\alpha \beta \gamma \delta} \psi_{\beta} \psi_{\gamma} \psi_{\delta}, \\
(\widetilde{X 7})_{\alpha \beta}= & -\frac{4}{k^{4}} \delta_{\alpha \beta} C_{\gamma \delta \epsilon} \psi_{0} \psi_{\gamma} \psi_{\delta}+\frac{6}{k^{4}}\left(C_{\alpha \gamma \delta} \psi_{0} \psi_{\beta} \psi_{\gamma} \psi_{\delta}+C_{\beta \gamma \delta} \psi_{0} \psi_{\alpha} \psi_{\gamma} \psi_{\delta}\right)+\frac{2}{3} C_{\gamma \delta \epsilon} \psi_{\gamma} \psi_{\delta} j_{\epsilon}- \\
& -\frac{1}{k^{3}}\left(C_{\alpha \gamma \delta} \psi_{\beta} \psi_{\gamma} j_{\delta}+C_{\beta \gamma \delta} \psi_{\alpha} \psi_{\gamma} j_{\delta}\right)-\frac{1}{k^{3}}\left(C_{\alpha \gamma \delta} \psi_{\gamma} \psi_{\delta} j_{\beta}+C_{\beta \gamma \delta} \psi_{\gamma} \psi_{\delta} j_{\alpha}\right)+ \\
& +\frac{11}{6 k^{4}} \delta_{\alpha \beta} C_{\gamma \delta \epsilon \zeta} \psi_{\gamma} \psi_{\delta} \psi_{\epsilon} \psi_{\zeta}-\frac{14}{3 k^{4}}\left(C_{\alpha \gamma \delta \epsilon} \psi_{\beta} \psi_{\gamma} \psi_{\delta} \psi_{\epsilon}+C_{\beta \gamma \delta \epsilon} \psi_{\alpha} \psi_{\gamma} \psi_{\delta} \psi_{\epsilon}\right), \\
(\widetilde{X 8})_{\alpha \beta}= & \frac{7}{3 k^{3}} \delta_{\alpha \beta} C_{\gamma \delta \epsilon} \psi_{\gamma} \psi_{\delta} \psi_{\epsilon}-2 C_{\beta \gamma \delta} \psi_{\alpha} \psi_{\gamma} \psi_{\delta}-5 C_{\alpha \gamma \delta} \psi_{\beta} \psi_{\gamma} \psi_{\delta}+C_{\alpha \beta \gamma \delta} \psi_{0} \psi_{\gamma} \psi_{\delta}+C_{\alpha \beta \gamma \delta} \psi_{\gamma} j_{\delta}, \\
(\widetilde{X 9})_{\alpha \beta}= & \frac{2}{k^{2}} C_{\alpha \beta \gamma \delta} \psi_{\gamma} \psi_{\delta} .
\end{aligned}
$$

Some comments are in order. The $k \rightarrow \infty$ limit is well-defined since the fields in (16) are of

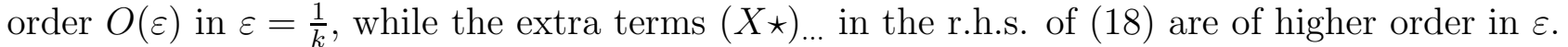
They are indeed of the order $O\left(\varepsilon^{2}\right)$ or higher. We could have normalized the fields (16) to be of order $O(1)$ in $\varepsilon$, but in this case the value of the central charge $c$ in the superconformal algebra would have been $\infty$. The only possibility of recovering a finite value for the superconformal central charge $c$ consists in performing the "classical renormalization" described above. The value of the conformal central charge $c$ is unrelated with $k$, the value of the affine central charge. The formula (18) presents for $c$ the value $c=-6$ ( $c$ is obtained as the Virasoro central charge from the first equation in (18) and corresponds to twelve times the coefficient of $\left.\delta^{\prime \prime \prime}\right)$. However, 
this value can be normalized at will (since we are dealing with classical Poisson brackets which satisfy by construction a graded version of the Leibniz property) through a simultaneous finite rescaling of both the fields (collectively denoted as $\left.\phi_{i}\right)$ in (14) $\left(\phi_{i} \mapsto z \phi_{i}\right)$ and the Poisson brackets (14) $\left(\{.,.\} \mapsto \frac{1}{z}\{.,\}.\right)$. It turns out that $c \mapsto z c$ and in particular we can set $c=1$ whenever is $c \neq 0$.

The closed superconformal algebra, recovered for $(X \star)_{\ldots} \equiv 0$, coincides with the so-called "Non-Associative Superconformal $N=8$ Algebra" introduced for the first time in reference [5].

The term "non-associative" is in reference with the fact that it does not satisfy a(super)Jacobi property. It is not even a (super)Malcev algebra. This point can be understood by noticing that, under the composition law $\mathbf{x}=\mathbf{x}_{1} \cdot \mathbf{x}_{2}$, the (super)Jacobi property is guaranteed for the triple $\mathbf{x}, \mathbf{y}, \mathbf{z}$, whenever (super)Jacobi is separately satisfied for the triples $\mathbf{x}_{1}, \mathbf{y}, \mathbf{z}$ and $\mathbf{x}_{2}, \mathbf{y}, \mathbf{z}\left(J\left(\mathbf{x}_{1}, \mathbf{y}, \mathbf{z}\right)=J\left(\mathbf{x}_{2}, \mathbf{y}, \mathbf{z}\right)=0 \Rightarrow J(\mathbf{x}, \mathbf{y}, \mathbf{z})=0\right)$. On the contrary, the (super)Malcev condition (6) is not automatically closed under such a composition law. Therefore the superMalcev property satisfied by the superaffine algebra $\widehat{\mathcal{O}}$ does not guarantee a super(Malcev) property for the superconformal algebra extracted through Sugawara construction. Indeed, an explicit counterexample can be given such that (6) is not verified. It is sufficient to take $\mathbf{x} \equiv J_{1}(x), \mathbf{y} \equiv Q_{4}(y), \mathbf{z} \equiv J_{2}(z)$

On the other hand the bosonic subalgebra, which is restricted to the fields $T(x)$ and $J_{\alpha}(x)$ alone, is a Malcev algebra.

The existence of the (16) Sugawara ensures that the superaffine algebra $\widehat{\mathcal{O}}$ is compatible with the global $N=8$ supersymmetry. This result is not surprising and would have been expected since the algebra is obtained in terms of the octonionic structure constants. In any case the existence of the (16) Sugawara allows us to explicitly compute the global $N=8$ transformations carried by the $j_{a}(x)$ and $\psi_{a}(x)$ fields entering (14). Indeed, let us introduce the $N=8$ global supersymmetric charges $\mathbf{Q}_{a}$ through

$$
\mathbf{Q}_{a}=\oint d x Q_{a}(x)
$$

where $a=0,1,2, \ldots, 7$ and $Q_{0}(x) \equiv Q(x)$. The supersymmetric transformation properties for the fields $j_{a}(x), \psi_{a}(x)$ are recovered from (14) and (16) after the $k \rightarrow \infty$ limit is taken into account. We have explicitly

$$
\begin{aligned}
\delta_{\epsilon_{0} \mathbf{Q}_{0}} \psi_{0} & =\epsilon_{0} \frac{j_{0}}{2} \\
\delta_{\epsilon_{0} \mathbf{Q}_{0}} j_{0} & =\epsilon_{0} \frac{\psi_{0}^{\prime}}{2} \\
\delta_{\epsilon_{0} \mathbf{Q}_{0}} \psi_{\alpha} & =\epsilon_{0} \frac{j_{\alpha}}{2} \\
\delta_{\epsilon_{0} \mathbf{Q}_{0}} j_{\alpha} & =\epsilon_{0}\left(\frac{\psi_{\alpha}^{\prime}}{2}-\frac{2}{k^{2}} C_{\alpha \beta \gamma \delta} \psi_{\beta} \psi_{\gamma} \psi_{\delta}\right) \\
\delta_{\epsilon_{\alpha} \mathbf{Q}_{\alpha}} \psi_{0} & =\epsilon_{\alpha} \frac{j_{\alpha}}{2} \\
\delta_{\epsilon_{\alpha} \mathbf{Q}_{\alpha}} j_{0} & =-\epsilon_{\alpha} \frac{\psi_{\alpha}^{\prime}}{2} \\
\delta_{\epsilon_{\alpha} \mathbf{Q}_{\alpha}} \psi_{\beta} & =\epsilon_{\alpha}\left(-\delta_{\alpha \beta} \frac{j_{0}}{2}-\frac{1}{2} C_{\alpha \beta \gamma} j_{\gamma}-\frac{1}{k}\left(\psi_{\alpha} \psi_{\beta}+C_{\alpha \beta \gamma} \psi_{0} \psi_{\gamma}\right)\right)
\end{aligned}
$$




$$
\begin{aligned}
\delta_{\epsilon_{\alpha} \mathbf{Q}_{\alpha} j_{\beta}=} & \epsilon_{\alpha}\left(\delta_{\alpha \beta} \frac{\psi_{0}^{\prime}}{2}+\frac{1}{2} C_{\alpha \beta \gamma} \psi_{\gamma}^{\prime}+\frac{1}{k}\left(C_{\alpha \beta \gamma} \psi_{\gamma} j_{0}-C_{\alpha \beta \gamma} \psi_{0} \psi_{\gamma}+\psi_{\alpha} j_{\beta}-\psi_{\beta} j_{\alpha}+C_{\alpha \beta \gamma \delta} \psi_{\gamma} \psi_{\delta}\right)+\right. \\
& \left.+\frac{2}{k^{2}}\left(\delta_{\alpha \beta} C_{\gamma \delta \epsilon} \psi_{\gamma} \psi_{\delta} \psi_{\epsilon}-C_{\beta \gamma \delta} \psi_{\alpha} \gamma \delta-2 C_{\alpha \gamma \delta} \psi_{\beta} \psi_{\gamma} \psi_{\delta}\right)\right) .
\end{aligned}
$$

The global supersymmetric transformations, dependent on the infinitesimal fermionic parameter $\epsilon_{a}$, are recovered from the above formulas after taking the $k \rightarrow \infty$ limit. Please notice that no summation is made for what concerns the index $\alpha$.

\section{Conclusions}

In this paper we have presented the generalization to the supersymmetric case of some results due to Osipov [2] concerning the bosonic Malcev affinization of the commutator algebra of the octonions and its Sugawara construction. We have introduced the superaffine Malcev algebra $\widehat{\mathcal{O}}$, realized by eight bosonic spin-1 and eight fermionic spin- $\frac{1}{2}$ fields, and have been able to prove that a Sugawara construction exists which maps, after a suitable limit is taken, these fields into the $N=8$ non-associative superconformal algebra of reference [5].

As a corollary, the superaffine algebra $\widehat{\mathcal{O}}$, besides being manifestly $N=1$ supersymmetric, is globally $N=8$ supersymmetric.

Such an algebraic construction can find a variety of applications (some of them are currently under investigation) to a full class of physical problems involving $N=8$ extended supersymmetries.

E.g. the superaffine $\widehat{\mathcal{O}}$ algebra defines a Poisson bracket structure underlining the $N=8$ supersymmetric extension of the $N L S-m K d V$ equations, while the Sugawara-induced nonassociative superconformal algebra gives the Poisson brackets for an $N=8$ extension of $\mathrm{KdV}$. This is the content of a forthcoming paper [7] which extends the construction of [4] to the present case.

Further topics of investigation concern the geometric approach (see [8]) to the classical superstring dynamics. It is expected that the motion of a classical superstring in a 10-dimensional target could be reduced to a octonionic valued superLiouville theory, which should be naturally described through the superalgebras introduced here. On the other hand, either WZNW-type models defined in the "almost group manifold $S^{7 "}$ (the almost being referred to the fact that it can be recovered from imaginary octonions, whose product however is non-associative) and the dynamics of generalized tops moving on $S^{7}$ are likely to be described by (super)-Malcev algebras. A converse approach, which retains Jacobi identities at the price of making fielddependent the algebraic structure constants (soft-algebras) has been suggested in [9]. Another natural field of investigation concerns the twistor formalism applied to the Green-Schwarz string (see e.g. [10]).

\section{References}

[1] T. Kugo and P. Townsend, Nucl. Phys. B 221 (1983) 357.

[2] E.P. Osipov, Phys. Lett. B 214 (1988) 371; Lett. Math. Phys. 18 (1989) 35.

[3] E.P. Osipov, Phys. Lett. B 274 (1992) 341.

[4] E. Ivanov, S. Krivonos and F. Toppan, Phys. Lett. B 405 (1997) 85. 
[5] F. Englert, A. Sevrin, W. Troost, A. van Proeyen and P. Spindel, J. Math. Phys. 29 (1988) 281.

[6] M. Günaydin and S.V. Ketov, Nucl. Phys. B 467 (1996) 215.

[7] H.L. Carrion, M. Rojas and F. Toppan, "Division Algebras and Extended $N=1,2,4,8$ KdVs", Preprint CBPF-NF-012/01.

[8] I. Bandos, D. Sorokin and D. Volkov, Phys. Lett. B 372 (1996) 77.

[9] M. Cederwall and C. Preitschopf, Comm. Math. Phys. 167 (1995) 373.

[10] N. Berkovits, Nucl. Phys. B 358 (1991) 169. 